PROCEEDINGS OF THE

AMERICAN MATHEMATICAL SOCIETY

Volume 126, Number 4, April 1998, Pages 1089-1099

S 0002-9939(98)04668-1

\title{
ON A THEOREM OF PICARD
}

\author{
F. GESZTESY AND W. STICKA
}

(Communicated by Hal L. Smith)

\begin{abstract}
We extend Picard's theorem on the existence of elliptic solutions of the second kind of linear homogeneous $n^{\text {th }}$-order scalar ordinary differential equations with coefficients being elliptic functions (associated with a common period lattice) to linear homogeneous first-order $n \times n$ systems. In particular, the qualitative Floquet-type structure of fundamental systems of solutions in terms of elliptic and exponential functions, polynomials, and Weierstrass zeta functions of the independent variable is determined. Connections with completely integrable systems are mentioned.
\end{abstract}

In order to set the stage for our extension of Picard's theorem on the existence of elliptic solutions of the second kind of $n^{\text {th }}$-order scalar ordinary differential equations with elliptic coefficients (with a common period lattice) to first-order $n \times n$ systems and an explicit description of the corresponding Floquet-type structure of fundamental systems of solutions, we briefly review Floquet theory for singly periodic $n \times n$ systems.

Denote by $\mathrm{M}(n), n \in \mathbb{N}$, the set of $n \times n$ matrices with entries in $\mathbb{C}$, define $\mathrm{GL}(n):=\{A \in \mathrm{M}(n) \mid \operatorname{det}(A) \neq 0\}$, and consider the linear homogeneous system

$$
\Psi^{\prime}(z)=Q(z) \Psi(z), \quad z \in \mathbb{C},
$$

where $\Psi(z) \in \mathrm{GL}(n), Q(z) \in \mathrm{M}(n)$, with $Q(z)$ a continuous periodic matrix of period $\Omega \in \mathbb{C} \backslash\{0\}$, that is,

$$
Q(z+\Omega)=Q(z), \quad z \in \mathbb{C} .
$$

Concerning (continuously differentiable) fundamental matrices $\Phi(z)$ of solutions of (1), one has the following basic Floquet theorem (see, e.g., [5], Ch. 3, [20], Ch. 4, [30], Ch. 5).

Theorem 1. Let $Q(z) \in \mathrm{M}(n)$ with $Q(z)$ a continuous periodic matrix of period $\Omega \in \mathbb{C} \backslash\{0\}$. Then (1) admits a fundamental matrix $\Phi(z) \in \mathrm{GL}(n)$ of the type

$$
\Phi(z)=P(z) \exp (z K), \quad z \in \mathbb{C},
$$

where $P(z) \in \mathrm{GL}(n), K \in \mathrm{M}(n)$, and $P(z)$ is continuously differentiable and periodic of period $\Omega$,

$$
P(z+\Omega)=P(z), \quad z \in \mathbb{C} .
$$

Received by the editors September 23, 1996.

1991 Mathematics Subject Classification. Primary 33E05, 34C25; Secondary 58F07.

The research was based upon work supported by the National Science Foundation under Grant No. DMS-9623121.

(C)1998 American Mathematical Society 
Moreover, linearly independent solutions $\underline{\phi}_{m}(z) \in \mathbb{C}^{n}, 1 \leq m \leq n$, of (1), that is, column vectors of (3), are of the type

$$
\underline{\phi}_{m}(z)=\sum_{k=0}^{n-1} \underline{p}_{m, k}(z) \exp \left(z \lambda_{m, k}\right) z^{k}
$$

$$
\underline{p}_{m, k}(z) \in \mathbb{C}^{n}, \quad \underline{p}_{m, k}(z+\Omega)=\underline{p}_{m, k}(z), \quad 1 \leq m \leq n, \quad 0 \leq k \leq n-1, \quad z \in \mathbb{C},
$$

where $\left\{\lambda_{m}\right\}_{1 \leq m \leq n}$ denotes a collection of all eigenvalues (not necessarily distinct) of $K$. In particular, there exists at least one solution $\underline{\phi}_{m_{0}}(z)$ of the form

$$
\underline{\phi}_{m_{0}}(z)=\underline{p}_{m_{0}, 0}(z) \exp \left(z \lambda_{m_{0}}\right)
$$

for some $m_{0} \in\{1, \ldots, n\}$ and, if $K$ is diagonalizable, all solutions $\underline{\phi}_{m}(z)$ are of this form, that is,

$$
\underline{\phi}_{m}(z)=\underline{p}_{m, 0}(z) \exp \left(z \lambda_{m, 0}\right), \quad 1 \leq m \leq n .
$$

More generally, associated with each distinct eigenvalue $\lambda_{r}$ of $K$ there exists at least one Floquet solution $\underline{\phi}_{r}(z)$ of the form $(7)$.

The existence of polynomials $z^{k}, k \geq 1$, in (5) is in a one-to-one correspondence with the nondiagonalizability of $K$ (i.e., its nontrivial Jordan form), or equivalently, with that of the monodromy matrix $M \in \mathrm{GL}(n)$ defined by

$$
M:=\Phi(\Omega)=\exp (\Omega K) .
$$

In particular, the bound

$$
k \leq n-1
$$

in (5) is an obvious consequence of the fact that

$$
e^{z J_{r}}=e^{z \lambda}\left(\begin{array}{cccccc}
1 & z & \frac{z^{2}}{2 !} & \cdots & \frac{z^{r-2}}{(r-2) !} & \frac{z^{r-1}}{(r-1) !} \\
0 & 1 & z & \cdots & \frac{z^{r-3}}{(r-3) !} & \frac{z^{r-2}}{(r-2) !} \\
\cdot & . & . & \cdots & \cdot & \cdot \\
\cdot & \cdot & \cdot & \cdots & . & \cdot \\
0 & 0 & 0 & \cdots & 1 & z \\
0 & 0 & 0 & \cdots & 0 & 1
\end{array}\right),
$$

for $r \times r$ Jordan blocks

$$
J_{r}=\left(\begin{array}{cccccc}
\lambda & 1 & 0 & \cdots & 0 & 0 \\
0 & \lambda & 1 & \cdots & 0 & 0 \\
\cdot & \cdot & \cdot & \cdots & \cdot & \cdot \\
. & . & . & \cdots & . & . \\
0 & 0 & 0 & \cdots & \lambda & 1 \\
0 & 0 & 0 & \cdots & 0 & \lambda
\end{array}\right) \in \mathrm{M}(r)
$$

Transforming $K$ into its Jordan normal form in (3) then yields a further wellknown simplification of the structure of the solutions in (5); we omit further details (see, e.g., [5], p. 80-81) since they will not be needed in this note.

Traditionally, (3) and (5) are called Floquet representations of the solutions of (1). In contrast to this general terminology we shall call $\underline{\phi}_{m_{0}}(z)$ a Floquet solution of (1) if the nontrivial polynomial part in (5) is absent, that is, if $\underline{\phi}_{m_{0}}$ is of the form 
in (6). In this case $\underline{\phi}_{m_{0}}(z)$ is reproduced up to a constant multiple if $z$ is replaced by $z+\Omega$,

$$
\underline{\phi}_{m_{0}}(z+\Omega)=\rho_{m_{0}} \underline{\phi}_{m_{0}}(z), \quad \rho_{m_{0}}=\exp \left\{\Omega \lambda_{m_{0}, 0}\right\} \in \mathbb{C} \backslash\{0\},
$$

and hence the eigenvalue $\rho_{m_{0}}$ of $M$ is called a Floquet multiplier.

Given these preliminaries we now turn to corresponding first-order systems with elliptic coefficients. Thus, we consider the homogeneous system (1) under the hypothesis that $Q(z) \in \mathrm{M}(n)$ is a nonconstant elliptic matrix, that is, $Q(z)$ is meromorphic on $\mathbb{C}$ and doubly periodic with period lattice $\Lambda$ spanned by $\Omega_{1}, \Omega_{3} \in \mathbb{C} \backslash\{0\}$, $\operatorname{Im}\left(\Omega_{1} / \Omega_{3}\right) \neq 0$,

$$
Q\left(z+\Omega_{j}\right)=Q(z), \quad j=1,3, \quad z \in \mathbb{C} .
$$

(Here we follow the usual terminology $\Omega_{1}, \Omega_{3}$ for the two periods, and denote $\Omega_{2}=\Omega_{1}+\Omega_{3}$ and $\Omega_{0}$ (or $\left.\Omega_{4}\right)=0$ such that $\Omega_{0}, \Omega_{1}, \Omega_{2}$, and $\Omega_{3}$ represent the vertices of the fundamental period parallelogram.)

Since $Q(z)$ is periodic with periods $\Omega_{1}$ and $\Omega_{3}$, one can apply Floquet theory, as described in Theorem 1, separately to the $\Omega_{1}$ and $\Omega_{3}$ directions. In particular, this yields fundamental matrices $\Phi_{j}(z) \in \mathrm{GL}(n), j=1,3$, of the type

$$
\Phi_{j}(z)=P_{j}(z) \exp \left(z K_{j}\right), \quad P_{j}\left(z+\Omega_{j}\right)=P_{j}(z), \quad j=1,3, \quad z \in \mathbb{C},
$$

assuming without loss of generality that $z=0$ is not a pole of $Q(z)$. Similarly, $M_{j} \in \mathrm{GL}(n), j=1,3$, denote the monodromy matrices

$$
M_{j}=\exp \left\{\Omega_{j} K_{j}\right\}, \quad j=1,3 .
$$

However, separate Floquet theory in the directions $\Omega_{1}$ and $\Omega_{3}$ is not at all what we have in mind, rather we would like to conclude the existence of some sort of Floquet representation (such as (3)) simultaneously for both directions $\Omega_{1}$ and $\Omega_{3}$. More precisely, we would hope to find some solutions $\underline{\phi}_{m_{0}}$ which are simultaneous Floquet solutions in the $\Omega_{1}$ and $\Omega_{3}$ directions, that is, solutions $\underline{\phi}_{m_{0}}(z) \in \mathbb{C}^{n}$ satisfying

$$
\underline{\phi}_{m_{0}}\left(z+\Omega_{j}\right)=\rho_{m_{0}, j} \underline{\phi}_{m_{0}}(z), \quad \rho_{m_{0}, j} \in \mathbb{C} \backslash\{0\}, \quad j=1,3,
$$

in analogy to (12), and, in general, a fundamental system of solutions extending (3) and (5) with $P(z)$ an elliptic matrix. As it turns out, the infinite set of poles of $Q(z)$ (being doubly periodic and nonconstant) renders this problem a formidable one due to the nontrivial underlying monodromy groups. Apparently, Picard, relying on previous work by Hermite, was the first to find a solution to this problem.

Studying linear homogeneous $n^{\text {th }}$-order scalar ordinary differential equations of the type

$$
\sum_{m=0}^{n} p_{m}(z) \psi^{(m)}(z)=0, \quad p_{n}(z)=1, \quad z \in \mathbb{C},
$$

with $\left\{p_{m}(z)\right\}_{0 \leq m \leq n-1}$ elliptic functions (not all constant to avoid trivialities) associated with the period lattice $\Lambda$ spanned by $\Omega_{j}, j=1,3$, he obtained the following result.

Theorem 2 (Picard [26], [27], [28], see also [2], p. 182-187, [22], p. 375-376). Assume that (17) has a meromorphic fundamental system of solutions. Then there 
exists at least one solution $\phi_{m_{0}}$ of (17) which is elliptic of the second kind, that is, $\phi_{m_{0}}(z)$ is meromorphic on $\mathbb{C}$ and

$$
\phi_{m_{0}}\left(z+\Omega_{j}\right)=\rho_{m_{0}, j} \phi_{m_{0}}(z), \quad j=1,3, \quad z \in \mathbb{C},
$$

for some $\rho_{m_{0}, j} \in \mathbb{C} \backslash\{0\}$. Moreover, if either $M_{1}$ or $M_{3}$ has distinct eigenvalues, then there exists a fundamental system $\phi_{m}(z), 1 \leq m \leq n$, of solutions of (17) which are all elliptic of the second kind,

$$
\phi_{m}\left(z+\Omega_{j}\right)=\rho_{m, j} \phi_{m}(z), \quad j=1,3, \quad 1 \leq m \leq n, \quad z \in \mathbb{C},
$$

for some $\rho_{m, j} \in \mathbb{C} \backslash\{0\}$.

Here, in obvious notation, $M_{j}, j=1,3$, denote the monodromy matrices obtained by rewriting (17) as a first-order system of the type (1).

A look at Theorem 2 reveals that Picard introduced the highly nontrivial assumption of the existence of a meromorphic fundamental system of (1) to enforce trivial monodromy properties of all solutions (which are encoded in the commutativity of $M_{1}$ and $M_{3}$, cf. the proof of Theorem 6). What we call Picard's theorem following the usual convention in [2], p. 182-185, [4], p. 338-343, [19], p. 536-539, and [23], p. 181-189, appears, however, to have a longer history. In fact, Picard's investigations [26], [27], [28] were inspired by earlier work of Hermite in the special case of Lamé's equation [21], p. 118-122, p. 266-418, p. 475-478 (see also [3], Sect. 3.6.4, and [29], p. 570-576). Further contributions were made by MittagLeffler [24], and Floquet [8], [9], [10]. (Curiously enough, Floquet seems to have worked on the doubly periodic case first and only then developed the singly periodic theory which now bears his name.) Detailed accounts of Picard's differential equation can be found in [19], p. 532-574, [23], p. 198-288. A typical example satisfying Picard's hypothesis of a meromorphic fundamental system of solutions is provided by the celebrated Lamé equation $\psi^{\prime \prime}(z)+[\lambda-g(g+1) \wp(z)] \psi(z)=0$, with $\wp(z)$ the elliptic Weierstrass function defined in (23), and $g \in \mathbb{Z}, \lambda \in \mathbb{C}$. See, for instance, [12] for a detailed discussion. More generally, it has recently been proven in [15] that $\psi^{\prime \prime}(z)+[\lambda+q(z)] \psi(z)=0$ satisfies Picard's hypothesis of the existence of a meromorphic fundamental system of solutions if and only if $q$ is a stationary solution of (at least) one of the equations of the Korteweg-de Vries hierarchy.

Even though Picard did mention certain extensions of his result to first-order systems (see, e.g., [18], p. 248-249), apparently he did not seek a Floquet-type representation for systems in the elliptic case. The first to study such a representation seems to have been Fedoryuk who proved the following result.

Theorem 3 (Fedoryuk [7]). Let $Q(z) \in \mathrm{M}(n)$ with $Q(z)$ an elliptic matrix of periods $\Omega_{j} \in \mathbb{C} \backslash\{0\}, j=1,3, \operatorname{Im}\left(\Omega_{1} / \Omega_{3}\right) \neq 0$, and suppose that (1) has a singlevalued fundamental matrix of solutions. Then (1) admits a fundamental matrix $\Phi(z) \in \mathrm{GL}(n)$ of the type

$$
\Phi(z)=D(z) \exp (z S+\zeta(z) T), \quad z \in \mathbb{C},
$$

where $S, T \in \mathrm{M}(n), D(z) \in \mathrm{GL}(n), D(z)$ is doubly periodic,

$$
D\left(z+\Omega_{j}\right)=D(z), \quad j=1,3, \quad z \in \mathbb{C},
$$


$\zeta(z)$ denotes the Weierstrass zeta function associated with the period lattice $\Lambda$ (cf. (24)), and

$$
S=\frac{1}{\pi i}\left[\Omega_{3} \zeta\left(\frac{\Omega_{1}}{2}\right) K_{3}-\Omega_{1} \zeta\left(\frac{\Omega_{3}}{2}\right) K_{1}\right], \quad T=-\frac{\Omega_{1} \Omega_{3}}{2 \pi i}\left(K_{3}-K_{1}\right) .
$$

Moreover, $K_{1}$ and $K_{3}$, and hence $S$ and $T$, commute.

Remark 4. Fedoryuk's representation (20) has the peculiar feature that it seems to stress an apparent essential singularity structure of solutions at $z=0$. Indeed, since $\zeta(z)$ has a first-order pole at $z=0$, the term $\exp (\zeta(z) T)$ in $(20)$ exhibits an essential singularity unless $T$ is nilpotent. Hence, the doubly periodic matrix $D(z)$, in general, will cancel this essential singularity of $\exp (\zeta(z) T)$ and therefore cannot be meromorphic, respectively, elliptic. Thus Fedoryuk's result cannot be considered the natural extension of Picard's Theorem 2.

In the remainder of this note we shall focus on an alternative to Theorem 3 and derive a generalization of Picard's theorem to first-order systems with elliptic coefficients which simultaneously describes the Floquet-type representation of the corresponding fundamental systems of solutions.

To facilitate matters we briefly discuss a few facts on the Weierstrass $\wp(z), \sigma(z)$, and $\zeta(z)$ functions associated with the period lattice $\Lambda$. The Weierstrass $\wp(z)$ function is defined by (cf. [1], Ch. 18)

$$
\wp(z):=z^{-2}+\sum_{(r, s) \in \mathbb{Z}^{2} \backslash\{(0,0)\}}\left[\left(z-r \Omega_{1}-s \Omega_{3}\right)^{-2}-\left(r \Omega_{1}+s \Omega_{3}\right)^{-2}\right] ;
$$

the Weierstrass zeta function $\zeta(z)$ by

$$
\zeta^{\prime}(z):=-\wp(z), \quad \lim _{z \rightarrow 0}\left[\zeta(z)-z^{-1}\right]=0 ;
$$

and the Weierstrass sigma function $\sigma(z)$ by

$$
\frac{\sigma^{\prime}(z)}{\sigma(z)}:=\zeta(z), \quad \lim _{z \rightarrow 0} \frac{\sigma(z)}{z}=1 .
$$

We will also need the Legendre relations,

$$
\begin{aligned}
\pi i & =\Omega_{3} \zeta\left(\Omega_{1} / 2\right)-\Omega_{1} \zeta\left(\Omega_{3} / 2\right) \\
& =\Omega_{2} \zeta\left(\Omega_{1} / 2\right)-\Omega_{1} \zeta\left(\Omega_{2} / 2\right) \\
& =\Omega_{3} \zeta\left(\Omega_{2} / 2\right)-\Omega_{2} \zeta\left(\Omega_{3} / 2\right)
\end{aligned}
$$

and the addition relations

$$
\begin{gathered}
\sigma\left(z+\Omega_{j}\right)=-\sigma(z) \exp \left\{2 \zeta\left(\Omega_{j} / 2\right)\left[z+\left(\Omega_{j} / 2\right)\right]\right\}, \quad 1 \leq j \leq 3, \\
\zeta\left(z+z_{0}\right)=\zeta(z)+\zeta\left(z_{0}\right)+2^{-1}\left[\wp^{\prime}(z)-\wp^{\prime}\left(z_{0}\right)\right]\left[\wp(z)-\wp\left(z_{0}\right)\right]^{-1}, \quad z_{0} \in \mathbb{C} .
\end{gathered}
$$

While $\wp(z)$ is elliptic with periods $\Omega_{j}, j=1,3, \zeta(z)$ is meromorphic on $\mathbb{C}$ and $\sigma(z)$ is entire. Moreover, $\wp(z)$ satisfies the differential equation

$$
\wp^{\prime \prime}(z)=6 \wp(z)^{2}-\left(g_{2} / 2\right), \quad g_{2}:=60 \prod_{(r, s) \in \mathbb{Z}^{2} \backslash\{(0,0)\}}\left(r \Omega_{1}+s \Omega_{3}\right)^{-4} .
$$

Since we shall also need a matrix generalization of (27), we state the following result $\left(I_{n} \in \mathrm{GL}(n)\right.$ denotes the identity matrix in $\left.\mathrm{M}(n)\right)$. 
Lemma 5. Let $A \in \mathrm{M}(n), j=1,3, z \in \mathbb{C}$. Then

$$
\sigma\left(\left(z+\Omega_{j}\right) I_{n}+A\right)=-\sigma\left(z I_{n}+A\right) \exp \left\{2 \zeta\left(\frac{\Omega_{j}}{2}\right)\left[\left(z+\frac{\Omega_{j}}{2}\right) I_{n}+A\right]\right\}
$$

Proof. If $A$ is diagonalizable, then (30) is obvious from (27). If $A$ is not diagonalizable, approximate $A$ by a sequence of diagonalizable matrices $\left\{A_{n}\right\} \underset{n \rightarrow \infty}{\longrightarrow} A$, or alternatively, replace $A$ by $A+w B$ for an appropriate $B \in \mathrm{M}(n)$ such that $A+w B$ is diagonalizable for $w$ in an open set $U \subset \mathbb{C}$ and analytically continue (with respect to $w$ ) to $w=0$.

Given these preparations our principal result then reads as follows.

Theorem 6. Let $Q(z) \in \mathrm{M}(n)$ with $Q(z)$ elliptic with periods $\Omega_{j} \in \mathbb{C} \backslash\{0\}, j=$ $1,3, \operatorname{Im}\left(\Omega_{1} / \Omega_{3}\right) \neq 0$, and suppose that (1) has a meromorphic fundamental matrix $\Psi(z) \in \mathrm{GL}(n)$ of solutions. Then (1) admits a fundamental matrix of the type

$$
\begin{aligned}
\Phi(z)= & E(z) \sigma(z)^{-1} \sigma\left(z I_{n}-\frac{\Omega_{1} \Omega_{3}}{2 \pi i}\left(K_{3}-K_{1}\right)\right) \\
& \times \exp \left\{\frac{z}{\pi i}\left[\Omega_{3} \zeta\left(\frac{\Omega_{1}}{2}\right) K_{3}-\Omega_{1} \zeta\left(\frac{\Omega_{3}}{2}\right) K_{1}\right]\right\}, \quad z \in \mathbb{C},
\end{aligned}
$$

where $E(z) \in \mathrm{GL}(n)$ is elliptic with periods $\Omega_{j}$,

$$
E\left(z+\Omega_{j}\right)=E(z), \quad j=1,3, \quad z \in \mathbb{C},
$$

and $M_{j}=\exp \left(\Omega_{j} K_{j}\right), j=1,3$, with $M_{1}, M_{3}$ and $K_{1}, K_{3}$ commuting matrices,

$$
\left[M_{1}, M_{3}\right]=0=\left[K_{1}, K_{3}\right] .
$$

Moreover, linearly independent solutions $\underline{\phi}_{m}(z) \in \mathbb{C}^{n}, 1 \leq m \leq n$, of (1), that is, column vectors of (31), are of the type

$$
\begin{gathered}
\underline{\phi}_{m}(z)=\sum_{k_{1}=0}^{n_{1}} \sum_{k_{2}=0}^{n_{2}} \underline{e}_{m, k_{1}, k_{2}}(z) \exp \left(z \mu_{m, k_{1}, k_{2}}\right) z^{k_{1}} \zeta(z)^{k_{2}}, \\
\underline{e}_{m, k_{1}, k_{2}}\left(z+\Omega_{j}\right)=\underline{e}_{m, k_{1}, k_{2}}(z), \quad z \in \mathbb{C}, \quad 1 \leq m \leq n, \quad 0 \leq k_{\ell} \leq n_{\ell}, \quad \ell=1,2,
\end{gathered}
$$

with the restriction

$$
n_{1}+n_{2} \leq n-1 .
$$

Here $\left\{\mu_{m, k_{1}, k_{2}}\right\}_{\substack{1 \leq m \leq n \\ 0 \leq k_{\ell} \leq n_{\ell}}}$ denotes a collection of all (not necessarily distinct) eigenvalues of $(1 / \pi i)\left[\Omega_{3} \zeta\left(\Omega_{1} / 2\right) K_{3}-\Omega_{1} \zeta\left(\Omega_{3} / 2\right) K_{1}\right]$. In particular, there exists at least one solution $\underline{\phi}_{m_{0}}(z) \in \mathbb{C}^{n}$ of (1) which is elliptic of the second kind, that is, $\underline{\phi}_{m_{0}}(z)$ is meromorphic on $\mathbb{C}$ and

$$
\underline{\phi}_{m_{0}}\left(z+\Omega_{j}\right)=\rho_{m_{0}, j} \underline{\phi}_{m_{0}}(z), \quad j=1,3, \quad z \in \mathbb{C}
$$

for some $\rho_{m_{0}, j}=\exp \left(\Omega_{j} \mu_{m_{0}, 0,0}\right) \in \mathbb{C} \backslash\{0\}, j=1$, 3. In addition, if all eigenvalues of $M_{1}$ or $M_{3}$ are distinct, then there exists a fundamental system of solutions $\left\{\underline{\phi}_{m}(z)\right\}_{1 \leq m \leq n}$ of (1) with all $\underline{\phi}_{m_{0}}(z)$ elliptic of the second kind, that is,

$$
\underline{\phi}_{m}\left(z+\Omega_{j}\right)=\rho_{m, j} \underline{\phi}_{m}(z), \quad j=1,3, \quad 1 \leq m \leq n, \quad z \in \mathbb{C},
$$

where $\rho_{m, j}=\exp \left(\Omega_{j} \mu_{m, 0,0}\right) \in \mathbb{C} \backslash\{0\}, j=1,3,1 \leq m \leq n$. 
Proof. For any fundamental matrix $\Psi(z) \in \mathrm{GL}(n)$ of (1) one infers

$$
\Psi\left(z+\Omega_{j}\right)=\Psi(z) M_{j}, \quad M_{j}=\exp \left(\Omega_{j} K_{j}\right), \quad j=1,3, \quad z \in \mathbb{C} .
$$

Together with our hypothesis of the existence of a meromorphic fundamental matrix $\widetilde{\Psi}(z)$ this yields

$$
\begin{aligned}
\widetilde{\Psi}\left(\left(z+\Omega_{1}\right)+\Omega_{3}\right) & =\widetilde{\Psi}\left(z+\Omega_{1}\right) M_{3}=\widetilde{\Psi}(z) M_{1} M_{3} \\
=\widetilde{\Psi}\left(\left(z+\Omega_{3}\right)+\Omega_{1}\right) & =\widetilde{\Psi}\left(z+\Omega_{3}\right) M_{1}=\widetilde{\Psi}(z) M_{3} M_{1}
\end{aligned}
$$

and hence (33). Next, define

$$
\begin{aligned}
E(z):= & \widetilde{\Psi}(z) \exp \left(-\frac{z}{\pi i}\left[\Omega_{3} \zeta\left(\frac{\Omega_{1}}{2}\right) K_{3}-\Omega_{1} \zeta\left(\frac{\Omega_{3}}{2}\right) K_{1}\right]\right) \\
& \times \sigma\left(z I_{n}-\frac{\Omega_{1} \Omega_{3}}{2 \pi i}\left(K_{3}-K_{1}\right)\right)^{-1} \sigma(z) .
\end{aligned}
$$

By hypothesis, $E(z)$ is meromorphic. One obtains

$$
\begin{aligned}
E\left(z+\Omega_{j}\right)= & \widetilde{\Psi}(z) M_{j} \exp \left(-\frac{z+\Omega_{j}}{\pi i}\left[\Omega_{3} \zeta\left(\frac{\Omega_{1}}{2}\right) K_{3}-\Omega_{1} \zeta\left(\frac{\Omega_{3}}{2}\right) K_{1}\right]\right) \\
& \times \sigma\left(\left(z+\Omega_{j}\right) I_{n}-\frac{\Omega_{1} \Omega_{3}}{2 \pi i}\left(K_{3}-K_{1}\right)\right)^{-1} \sigma\left(z+\Omega_{j}\right) \\
= & \widetilde{\Psi}(z) \exp \left(-\frac{z}{\pi i}\left[\Omega_{3} \zeta\left(\frac{\Omega_{1}}{2}\right) K_{3}-\Omega_{1} \zeta\left(\frac{\Omega_{3}}{2}\right) K_{1}\right]\right) \\
& \times\left\{\exp \left(\Omega_{j} K_{j}\right) \exp \left(-\frac{\Omega_{j} \Omega_{3}}{\pi i} \zeta\left(\frac{\Omega_{1}}{2}\right) K_{3}\right)\right. \\
& \left.\times \exp \left(\frac{\Omega_{j} \Omega_{1}}{\pi i} \zeta\left(\frac{\Omega_{3}}{2}\right) K_{1}\right) \exp \left(\frac{\Omega_{1} \Omega_{3}}{\pi i} \zeta\left(\frac{\Omega_{j}}{2}\right)\left(K_{3}-K_{1}\right)\right)\right\} \\
& \times \sigma\left(z I_{n}-\frac{\Omega_{1} \Omega_{3}}{2 \pi i}\left(K_{3}-K_{1}\right)\right)^{-1} \sigma(z) \\
= & \widetilde{\Psi}(z) \exp \left(-\frac{z}{\pi i}\left[\Omega_{3} \zeta\left(\frac{\Omega_{1}}{2}\right) K_{3}-\Omega_{1} \zeta\left(\frac{\Omega_{3}}{2}\right) K_{1}\right]\right) \\
& \times \sigma\left(z I_{n}-\frac{\Omega_{1} \Omega_{3}}{2 \pi i}\left(K_{3}-K_{1}\right)\right)^{-1} \sigma(z) \\
= & E(z), \quad j=1,3
\end{aligned}
$$

using (38), Lemma 5 , the commutativity of $K_{1}$ and $K_{3}$, and the Legendre relations (26) (which yield $\{\cdots\}=I_{n}$ in (41)). Thus, $E(z)$ is elliptic, proving (31) and (32). Next, let $L_{1}, L_{2} \in \mathrm{M}(n),\left[L_{1}, L_{2}\right]=0$ and consider

$$
\begin{aligned}
\exp & \left(z_{1} L_{1}\right) \exp \left(z_{2} L_{2}\right) \\
= & \sum_{k_{1}=0}^{n_{1}} \sum_{k_{2}=0}^{n_{2}} H_{k_{1}, k_{2}} \exp \left(z_{1} \lambda_{1, k_{1}}+z_{2} \lambda_{2, k_{2}}\right) z_{1}^{k_{1}} z_{2}^{k_{2}}, \quad z_{1}, z_{2} \in \mathbb{C},
\end{aligned}
$$

where $\left\{\lambda_{p, k_{p}}\right\}_{0 \leq k_{p} \leq n_{p}}$ denotes a collection of all eigenvalues (not necessarily distinct) of $L_{p}, p=1,2$, and $H_{k_{1}, k_{2}} \in \mathrm{M}(n)$. Then

$$
n_{1}+n_{2} \leq n-1 \text {. }
$$


Indeed, assume the contrary, that is, suppose that (42) contains a term of the type $H_{k_{1}, k_{2}} z_{1}^{k_{1}} z_{2}^{k_{2}}$ with $k_{1}+k_{2} \geq n$. Then taking $z_{1}=z_{2}:=z$ in (42) and noting

$$
\exp \left(z L_{1}\right) \exp \left(z L_{2}\right)=\exp \left(z\left(L_{1}+L_{2}\right)\right)
$$

immediately yields a contradiction upon transforming $\left(L_{1}+L_{2}\right)$ into its Jordan normal form since (10) permits at most terms of degree $z^{n-1}$ in (44). Since for $\lambda \in \mathbb{C}$,

$$
\zeta\left(z-\frac{\Omega_{1} \Omega_{3}}{2 \pi i} \lambda\right)=\zeta(z)+\text { elliptic }
$$

by (28), a typical Jordan block associated with $\sigma\left(z I_{n}-\left(\Omega_{1} \Omega_{3} / 2 \pi i\right)\left(K_{3}-K_{1}\right)\right)$ is of the type

$$
\sigma\left(z-\frac{\Omega_{1} \Omega_{3}}{2 \pi i} \lambda\right)\left(\begin{array}{cccccc}
1 & a_{1}(z) & a_{2}(z) & \cdots & a_{r-2}(z) & a_{r-1}(z) \\
0 & 1 & a_{1}(z) & \cdots & a_{r-3}(z) & a_{r-2}(z) \\
\cdot & \cdot & \cdot & \cdots & \cdot & \cdot \\
\cdot & \cdot & \cdot & \cdots & \cdot & \cdot \\
0 & 0 & 0 & \cdots & 1 & a_{1}(z) \\
0 & 0 & 0 & \cdots & 0 & 1
\end{array}\right), \quad r \leq n
$$

where

$$
\begin{aligned}
a_{s}(z) & =\sum_{\ell=0}^{s} b_{\ell}(z) \zeta\left(z-\frac{\Omega_{1} \Omega_{3}}{2 \pi i} \lambda\right)^{\ell} \\
& =\sum_{\ell=0}^{s} c_{\ell}(z) \zeta(z)^{\ell}, \quad b_{s}(z)=c_{s}(z)=1, \quad b_{s-1}(z)=0, \quad 1 \leq s \leq r-1,
\end{aligned}
$$

with $b_{\ell}(z), c_{\ell}(z), 0 \leq \ell \leq s$, elliptic functions and $\lambda$ an eigenvalue of $\left(K_{3}-K_{1}\right)$. In order to arrive at (47), we used (cf. (24) and (25))

$$
\sigma^{(s)}(z)=\sigma(z) \sum_{\ell=0}^{s} b_{\ell}(z) \zeta(z)^{\ell}, \quad b_{s}(z)=1, \quad b_{s-1}(z)=0
$$

together with

$$
f\left(J_{r}\right)=\left(\begin{array}{ccccc}
f(\lambda) & \frac{f^{\prime}(\lambda)}{1 !} & \cdots & \frac{f^{(r-2)}(\lambda)}{(r-2) !} & \frac{f^{(r-1)}(\lambda)}{(r-1) !} \\
0 & f(\lambda) & \cdots & \frac{f^{(r-3)}(\lambda)}{(r-3) !} & \frac{f^{(r-2)}(\lambda)}{(r-2) !} \\
\cdot & \cdot & \cdots & \cdot & \cdot \\
0 & 0 & \cdots & f(\lambda) & \frac{f^{\prime}(\lambda)}{1 !} \\
0 & 0 & \cdots & 0 & f(\lambda)
\end{array}\right)
$$

for appropriate functions $f$ (cf. (11)) in the first step and (45) in the second step. Moreover, define

$$
\alpha(z)=\frac{\sigma\left(z-\frac{\Omega_{1} \Omega_{3}}{2 \pi i} \lambda\right)}{\sigma(z)}
$$

then (27) implies

$$
\alpha\left(z+\Omega_{j}\right)=\alpha(z) \exp \left\{\frac{\Omega_{1} \Omega_{3}}{\pi i} \zeta\left(\frac{\Omega_{j}}{2}\right) \lambda\right\}, \quad j=1,3 .
$$


Consequently, (31), (32), (46), (47), and (51) prove (34). Since $\zeta(z)^{s}$ is the leading power in $\zeta$ in $a_{s}$, in order to prove the bound (35) it suffices to replace

$$
\begin{gathered}
\sigma(z)^{-1} \sigma\left(z I_{n}-\frac{\Omega_{1} \Omega_{3}}{2 \pi i}\left(K_{3}-K_{1}\right)\right) \exp \left\{\frac{z}{\pi i}\left[\Omega_{3} \zeta\left(\frac{\Omega_{1}}{2}\right) K_{3}-\Omega_{1} \zeta\left(\frac{\Omega_{3}}{2}\right) K_{1}\right]\right\} \\
\longrightarrow \exp \left(\zeta(z) N_{3,1}\right) \exp \left(\frac{z}{\pi i}\left[\Omega_{3} \zeta\left(\frac{\Omega_{1}}{2}\right) K_{3}-\Omega_{1} \zeta\left(\frac{\Omega_{3}}{2}\right) K_{1}\right]\right)
\end{gathered}
$$

with a nilpotent matrix $N_{3,1} \in \mathrm{M}(n)$ whose Jordan block structure coincides with that of $\left(K_{3}-K_{1}\right)$. The matrix $N_{3,1}$ needs to be nilpotent so that its only eigenvalue zero avoids exponential terms of the type $\exp [\zeta(z) \lambda]$ in $\exp \left[\zeta(z) N_{3,1}\right]$ (cf. (10), (11)), since (46) displays no such exponential terms. An application of (42) and (43) then yields (35). Next, suppose $V_{3,1} \in \mathrm{GL}(n)$ transforms $\left(K_{3}-K_{1}\right)$ into its Jordan normal form $\widehat{K}_{3,1}$ (resp. $W_{1} \in \mathrm{GL}(n)$ transforms $K_{1}$ into its Jordan form $\left.\widehat{K}_{1}\right)$,

$$
\widehat{K}_{3,1}=V_{3,1}^{-1}\left(K_{3}-K_{1}\right) V_{3,1}, \quad \widehat{K}_{1}=W_{1}^{-1} K_{1} W_{1} .
$$

Then we may rewrite $\Phi(z)$ in (31) as

$$
\begin{aligned}
\Phi(z)= & \widehat{E}(z) \sigma(z)^{-1} \sigma\left(z I_{n}-\frac{\Omega_{1} \Omega_{3}}{2 \pi i} \widehat{K}_{3,1}\right) \\
& \times \exp \left[\frac{z}{\pi i} \Omega_{3} \zeta\left(\frac{\Omega_{1}}{2}\right) \widehat{K}_{3,1}\right] V_{3,1}^{-1} W_{1} \exp \left[z \widehat{K}_{1}\right] W_{1}^{-1}, \quad \widehat{E}(z)=E(z) V_{3,1}
\end{aligned}
$$

Applying (46) and (10) to the Jordan blocks of $\sigma\left(z I_{n}-\left(\Omega_{1} \Omega_{3} / 2 \pi i\right) \widehat{K}_{3,1}\right)$ and $\exp \left(z \widehat{K}_{1}\right)$ yields the existence of at least one solution $\phi_{m_{0}}(z) \in \mathbb{C}^{n}$ of (1) which is elliptic of the second kind and hence satisfies (36). Finally, if all eigenvalues of $M_{1}$ (or $M_{3}$ ) are distinct, then $M_{1}$ and $M_{3}$ (being commuting matrices) can simultaneously be put into Jordan normal form, that is, we can choose $V_{3,1}=W_{1}$ in (53) and (54) with both $\widehat{K}_{3,1}$ and $\widehat{K}_{1}$ being diagonal matrices (see, e.g., [11], p. 223-224). Hence, neither $\zeta(z)$ nor $z$ terms will arise in $\Phi(z)$ proving the last assertion (37).

We conclude with a series of remarks.

Remark 7. (i) If $M_{1}$ or $M_{3}$ has distinct eigenvalues, then, as a consequence of equation (54) with $V_{3,1}=W_{1}$, equation (1) has a fundamental system of solutions $\underline{\phi}_{m}(z) \in \mathbb{C}^{n}$ of the form

$$
\begin{aligned}
\underline{\phi}_{m}(z)= & \underline{e}_{m}(z) \sigma(z)^{-1} \sigma\left(z-\frac{\Omega_{1} \Omega_{3}}{2 \pi i} k_{3,1, m}\right) \\
& \times \exp \left[\frac{z}{\pi i} \Omega_{3} \zeta\left(\frac{\Omega_{1}}{2}\right) k_{3,1, m}\right] \exp \left[z k_{1, m}\right], \quad 1 \leq m \leq n, \quad z \in \mathbb{C},
\end{aligned}
$$

where $\underline{e}_{m}(z)$ are elliptic with period lattice $\Lambda$,

$$
\underline{e}_{m}\left(z+\Omega_{j}\right)=\underline{e}_{m}(z), \quad j=1,3, \quad 1 \leq m \leq n, \quad z \in \mathbb{C},
$$

and $\left\{k_{3,1, m}\right\}_{1 \leq m \leq n}$ and $\left\{k_{1, m}\right\}_{1 \leq m \leq n}$ are the eigenvalues of $\left(K_{3}-K_{1}\right)$ and $K_{1}$, respectively. 
(ii) In connection with equation (14) one computes

$$
\begin{aligned}
E(z)= & P_{j}(z) \sigma(z) \sigma\left(z I_{n}-\frac{\Omega_{1} \Omega_{3}}{2 \pi i}\left(K_{3}-K_{1}\right)\right)^{-1} \\
& \times \exp \left\{-\frac{z}{\pi i} \Omega_{r(j)} \zeta\left(\frac{\Omega_{r(j)}}{2}\right)\left(K_{3}-K_{1}\right)\right\}, \quad r(j)=\left\{\begin{array}{ll}
1, & j=3, \\
3, & j=1,
\end{array} \quad z \in \mathbb{C} .\right.
\end{aligned}
$$

(iii) In the special scalar case (17), the bound (35) is proven in [22], p. 377-378, for $n=2$ and stated (without proof) for $n \geq 3$.

(iv) We might note that it is not necessary to assume that $Q(z)$ is elliptic in Theorem 6. Indeed, if $Q(z)$ is merely doubly periodic, then the assumption of a meromorphic fundamental matrix $\Psi(z)$ of solutions of equation (1) then yields that $Q(z)=\Psi^{\prime}(z) \Psi(z)^{-1}$ is meromorphic and hence elliptic also. It is the additional assumption of a meromorphic fundamental matrix which allows one to go beyond Fedoryuk's Theorem 3.

(v) It seems worthwhile to note that the highly nontrivial assumption of the existence of a meromorphic fundamental system of solutions of equation (1) in Theorems 2 and 6 appears to be intimately connected with certain infinite-dimensional completely integrable Hamiltonian systems arising in the context of soliton equations (see, e.g., [3], [6], [25]). In fact, in a recent series of papers [12]-[16], the assumption of a meromorphic fundamental system of solutions in the scalar case $n=2$ in (17) led to an explicit characterization of all elliptic solutions of the stationary Korteweg-de Vries (KdV) hierarchy. These papers can be consulted for a variety of explicit examples satisfying the hypothesis of a meromorphic fundamental system of solutions. We expect that Theorem 6 will lead to an analogous characterization of all elliptic solutions of matrix hierarchies of soliton equations in-

cluding the Gelfand-Dickey hierarchy (see, e.g., [3], [6]). Quite recently, all elliptic solutions of the AKNS hierarchy were characterized in this manner in [17].

\section{REFERENCES}

[1] M. Abramowitz and I. A. Stegun, Handbook of Mathematical Functions, Dover, New York, 1972. MR 94b:00012 (reprint of 1972 edition)

[2] N. I. Akhiezer, Elements of the Theory of Elliptic Functions, Amer. Math. Soc., Providence, RI, 1990. MR 91k:33016

[3] E. D. Belokolos, A. I. Bobenko, V. Z. Enol'skii, A. R. Its, and V. B. Matveev, AlgebroGeometric Approach to Nonlinear Integrable Equations, Springer, Berlin, 1994.

[4] H. Burkhardt, Elliptische Funktionen, 2nd ed., Verlag von Veit, Leipzig, 1906.

[5] E. A. Coddington and N. Levinson, Theory of Ordinary Differential Equations, Krieger, Malabar, 1985.

[6] L. A. Dickey, Soliton Equations and Hamiltonian Systems, World Scientific, Singapore, 1991. MR 93d:58067

[7] M. V. Fedoryuk, Lamé wave functions in the Jacobi form, J. Diff. Eqs., 23, 1170-1177 (1987). MR 89c:33014

[8] G. Floquet, Sur les équations différentielles linéaires à coefficients doublement périodiques, C. R. Acad. Sci. Paris, 98, 38-39, 82-85 (1884).

[9] G. Floquet, Sur les équations différentielles linéaires à coefficients doublement périodiques, Ann. Sci. Ecole Norm. Sup., 1, 181-238 (1884).

[10] G. Floquet, Addition a un mémorie sur les équations différentielles linéaires, Ann. Sci. Ecole Norm. Sup., 1, 405-408 (1884).

[11] F. R. Gantmacher, Theory of Matrices, Vol. 1, Chelsea, New York, 1960. MR 21:6372c 
[12] F. Gesztesy and R. Weikard, Lamé potentials and the stationary ( $m) K d V$ hierarchy, Math. Nachr., 176, 73-91 (1995). CMP 96:04

[13] F. Gesztesy and R. Weikard, On Picard potentials, Diff. Int. Eqs., 8, 1453-1476 (1995). MR 96e:34141

[14] F. Gesztesy and R. Weikard, Treibich-Verdier potentials and the stationary $(m)$ KdV hierarchy, Math. Z. 219, 451-476 (1995). MR 96e:14030

[15] F. Gesztesy and R. Weikard, Picard potentials and Hill's equation on a torus, Acta Math., 176, 73-107 (1996). MR 97f:14046

[16] F. Gesztesy and R. Weikard, Toward a characterization of elliptic solutions of hierarchies of soliton equations, Contemp. Math., to appear.

[17] F. Gesztesy and R. Weikard, A characterization of all elliptic solutions of the AKNS hierarchy, Acta Math., to appear.

[18] J. Gray, Linear Differential Equations and Group Theory from Riemann to Poincaré, Birkhäuser, Boston, 1986. MR 89d:01041

[19] G.-H. Halphen, Traité des Fonctions Elliptiques, tome 2, Gauthier-Villars, Paris, 1888.

[20] P. Hartman, Ordinary Differential Equations, 2nd, ed., Birkhäuser, Boston, 1982. MR 83e:34002

[21] C. Hermite, Oeuvres, tome 3, Gauthier-Villars, Paris, 1912.

[22] E. L. Ince, Ordinary Differential Equations, Dover, New York, 1956. MR 6:65f

[23] M. Krause, Theorie der doppeltperiodischen Funktionen einer veränderlichen Grösse, Vol. 2, Teubner, Leipzig, 1897.

[24] G. Mittag-Leffler, Sur les équations différentielles linéaires à coefficients doublement périodiques, C. R. Acad. Sci. Paris, 90, 299-300 (1880).

[25] S. Novikov, S. V. Manakov, L. P. Pitaevskii, and V. E. Zakharov, Theory of Solitons, Consultants Bureau, New York, 1984. MR 86k:35142

[26] E. Picard, Sur une généralisation des fonctions périodiques et sur certaines équations différentielles linéaires, C. R. Acad. Sci. Paris, 89, 140-144 (1879).

[27] E. Picard, Sur une classe d'équations différentielles linéaires, C. R. Acad. Sci. Paris, 90, 128-131 (1880).

[28] E. Picard, Sur les équations différentielles linéaires à coefficients doublement périodiques, J. Reine Angew. Math., 90, 281-302 (1881).

[29] E. T. Whittaker and G. N. Watson, A Course of Modern Analysis, Cambridge University Press, Cambridge, 1996. MR 97k:01072

[30] V. A. Yakubovich and V. M. Starzhinskii, Linear Differential Equations with Periodic Coefficients, Vol. 1, Wiley, New York, 1975. MR 51:994

Department of Mathematics, University of Missouri, Columbia, Missouri 65211

E-mail address, F. Gesztesy: fritz@math.missouri.edu 